A Pardanani ${ }^{1}$, T Lasho $^{1}$, D Chen ${ }^{2}$, TK Kimlinger ${ }^{1}$, C Finke $^{1}$ D Zblewski ${ }^{1}$, MM Patnaik ${ }^{1}$, KK Reichard $^{2}$, E Rowinsky $^{3}$, CA Hanson $^{2}$, C Brooks ${ }^{3}$ and A Tefferi ${ }^{1}$

${ }^{1}$ Division of Hematology and Department of Medicine, Rochester, MN, USA;

${ }^{2}$ Division of Hematopathology and Department of Laboratory Medicine and Pathology, Mayo Clinic, Rochester, MN, USA and

${ }^{3}$ Stemline Therapeutics Inc., New York, NY, USA E-mail: Pardanani.animesh@mayo.edu

\section{REFERENCES}

1 Woodcock JM, Bagley CJ, Zacharakis B, Lopez AF. A single tyrosine residue in the membrane-proximal domain of the granulocyte-macrophage colony-stimulating factor, interleukin (IL)-3, and IL-5 receptor common beta-chain is necessary and sufficient for high affinity binding and signaling by all three ligands. J Biol Chem 1996; 271: 25999-26006.

2 Metcalf D, Begley CG, Johnson GR, Nicola NA, Lopez AF, Williamson DJ. Effects of purified bacterially synthesized murine multi-CSF (IL-3) on hematopoiesis in normal adult mice. Blood 1986; 68: 46-57.

3 Testa U, Pelosi E, Frankel A. CD 123 is a membrane biomarker and a therapeutic target in hematologic malignancies. Biomark Res 2014; 2: 4.

4 Jordan CT, Upchurch D, Szilvassy SJ, Guzman ML, Howard DS, Pettigrew AL et al. The interleukin-3 receptor alpha chain is a unique marker for human acute myelogenous leukemia stem cells. Leukemia 2000; 14: 1777-1784.

5 Florian S, Sonneck K, Hauswirth AW, Krauth MT, Schernthaner GH, Sperr WR et al. Detection of molecular targets on the surface of CD34+/CD38 - stem cells in various myeloid malignancies. Leuk Lymphoma 2006; 47: 207-222.

6 Frolova O, Benito J, Brooks C, Wang RY, Korchin B, Rowinsky EK et al. SL-401 and SL-501, targeted therapeutics directed at the interleukin-3 receptor, inhibit the growth of leukaemic cells and stem cells in advanced phase chronic myeloid leukaemia. Br J Haematol 2014; 166: 862-874.
7 He SZ, Busfield S, Ritchie DS, Hertzberg MS, Durrant S, Lewis ID et al. A phase 1 study of the safety, pharmacokinetics, and anti-leukemic activity of the anti-CD123 monoclonal antibody, CSL360, in relapsed, refractory or high-risk acute myeloid leukemia (AML). Leuk Lymphoma 2014; e-pub ahead of print 20 November 2014; doi:10.3109/10428194.2014.956316.

8 Frankel AE, Konopleva M, Hogge D, Rizzieri D, Brooks C, Cirrito $T$ et al. Activity and tolerability of SL-401, a targeted therapy directed to the interleukin-3 receptor on cancer stem cells and tumor bulk, as a single agent in patients with advanced hematologic malignancies. J Clin Oncol 2013; 31: 15.

9 Frankel AE, Woo JH, Ahn C, Pemmaraju N, Medeiros BC, Carraway HE et al. Activity of SL-401, a targeted therapy directed to interleukin-3 receptor, in blastic plasmacytoid dendritic cell neoplasm patients. Blood 2014; 124: 385-392.

10 Valent P, Besemer J, Sillaber C, Butterfield JH, Eher R, Majdic O et al. Failure to detect IL-3-binding sites on human mast cells. J Immunol 1990; 145: 3432-3437.

11 Teodosio C, Garcia-Montero AC, Jara-Acevedo M, Sanchez-Munoz L, Alvarez-Twose I, Nunez $\mathrm{R}$ et al. Mast cells from different molecular and prognostic subtypes of systemic mastocytosis display distinct immunophenotypes. J Allergy Clin Immunol 2010; 125: 719-726.

12 Moonim MT, Kossier T, van Der Walt J, Wilkins B, Harrison CN, Radia DH CD30/CD123 expression in systemic mastocytosis does not correlate with aggressive disease. Blood 2012; 120: 21.

13 Horny HP, Metcalfe DD, Bennett JM, Bain BJ, Akin C, Escribano L et al. Mastocytosis. In: Swerdlow $\mathrm{SH}$, Campo E, Harris NL, Jaffe ES, Pileri SA, Stein $\mathrm{H}$ et al. (eds) WHO Classification of Tumors of Hematopoietic and Lymphoid Tissues, 4th edn. International Agency for Research and Cancer (IARC): Lyon, 2008; pp 54-63.

14 Pardanani A. Systemic mastocytosis in adults: 2013 update on diagnosis, risk stratification, and management. Am J Hematol 2013; 88: 612-624.

15 Gotlib J, Kluin-Nelemans HC, George Tl, Akin C, Sotlar K, Hermine O et al. Durable responses and improved quality of life with midostaurin (PKC412) in advanced systemic mastocytosis (SM): updated stage 1 results of the Global D2201 trial. Blood 2013; 122: 21.

\title{
Quantifying ultra-rare pre-leukemic clones via targeted error-corrected sequencing
}

\section{Leukemia (2015) 29, 1608-1611; doi:10.1038/leu.2015.17}

The quantification of rare clonal and subclonal populations from a heterogeneous DNA sample has multiple clinical and research applications for the study and treatment of leukemia. Specifically, in the hematopoietic compartment, recent reports demonstrate the presence of subclonal variation in normal and malignant hematopoiesis, ${ }^{1,2}$ and leukemia is now recognized as an oligoclonal disease. $^{3}$ Currently, clonal heterogeneity in leukemia is studied using next-generation sequencing (NGS) targeting subclone-specific mutations. With this method, detecting mutations at $2-5 \%$ variant allele fraction (VAF) requires costly and time-intensive deep resequencing and identifying lower frequency variants is impractical regardless of sequencing depth. Recently, various methods have been developed to circumvent the error rate of NGS., ${ }^{4,5}$ These methods tag individual DNA molecules with unique oligonucleotide indexes, which enable error correction after sequencing.

Here we present a direct application of error-corrected sequencing (ECS) to study clonal heterogeneity during leukemogenesis and validate the accuracy of this method with a series

Figure 1. Benchmarking for ECS and the identification of rare pre-leukemic mutations. (a, b) DNA extracted from a diagnostic leukemia sample with known mutations in $R U N X 1$ (a) and IDH2 (b) was serially diluted into non-cancer, unrelated human DNA. Two replicates were run per sample/dilution. The coefficient of determination $\left(r^{2}\right)$ between diluted tumor concentration in the sample and VAF in the generated read families was 0.9999 and 0.9991 for $R U N X 1$ and $I D H 2$, respectively. (c) The VAF at every nucleotide not expected to contain mutations in the dilution series experiment were analyzed to determine the error profile of the error-corrected consensus sequences compared with conventional deep sequencing. A cumulative distribution function of VAF demonstrated a reduced error profile in read families relative to conventional deep sequenced reads. (d) The most frequent class of substitution seen in read families was in $G$ to $T(C$ to $A)$ transversions, which was consistent with oxidative conversion of guanine to 8-oxo-guanine. (e, f) The leukemia-specific variants identified in ASXL1 and U2AF1 at diagnosis (circled) were not distinguishable from sequencing errors in the same substitution class by conventional deep sequencing. $(\mathbf{g}, \mathbf{h})$ Targeted error-corrected sequencing identified the ASXL1 variant in the 2002 banked sample at 0.004 VAF and the U2AF1 variant in the 2004 banked sample at 0.009 VAF. 
a

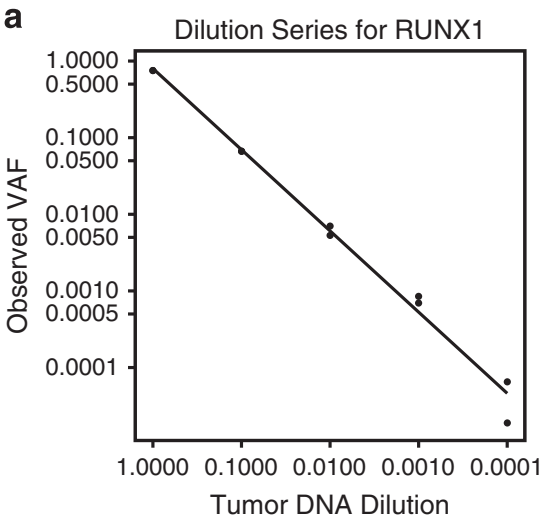

C

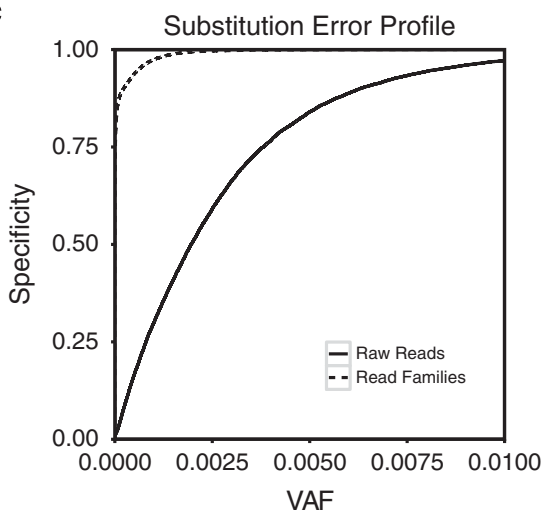

e

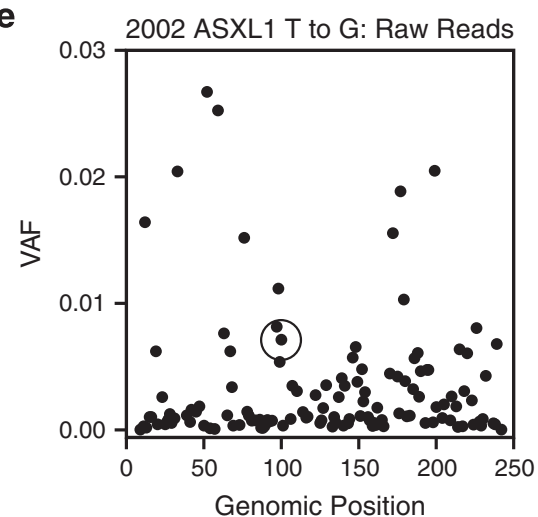

g

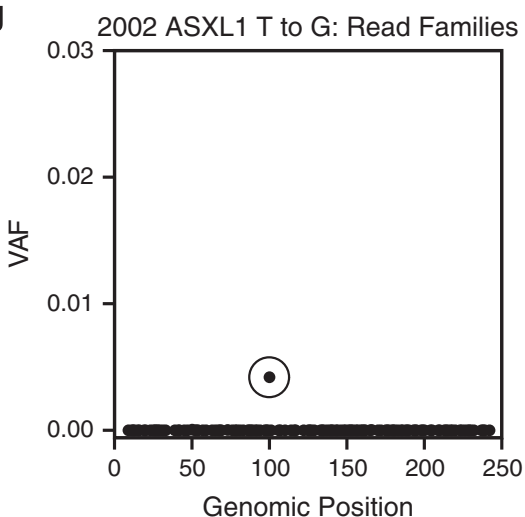

b

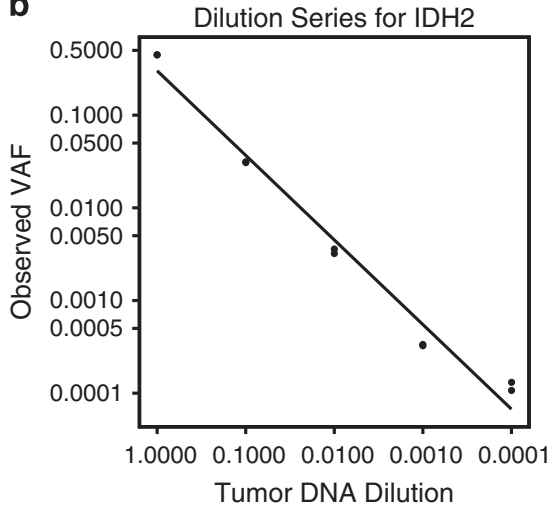

d

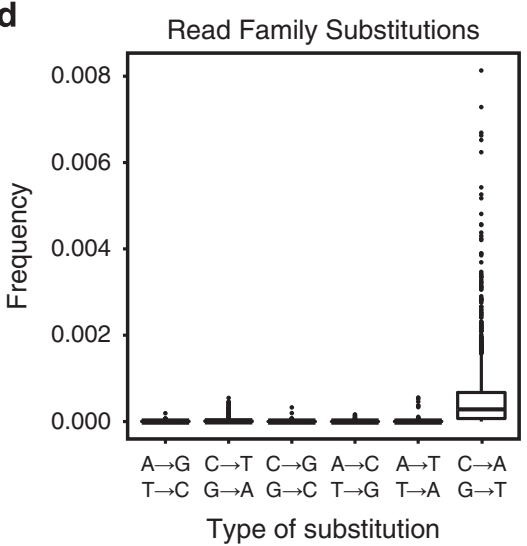

f

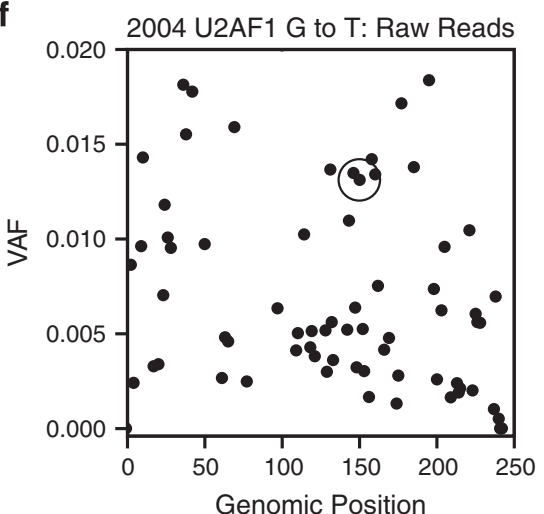

h

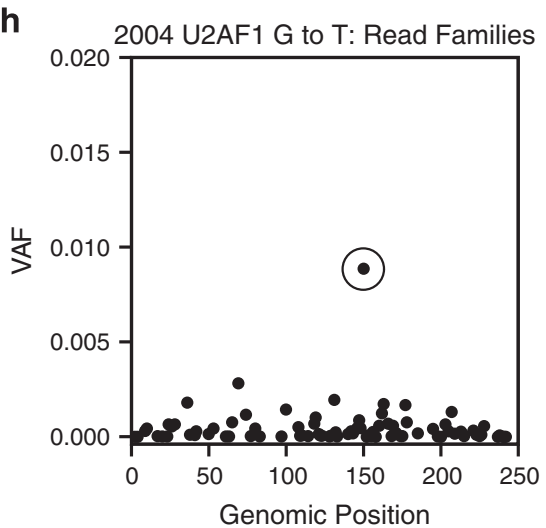


of benchmarking experiments. Specifically, we demonstrate the ability of ECS to identify leukemia-associated mutations in banked pre-leukemic blood and bone marrow from patients with either therapy-related acute myeloid leukemia (t-AML) or therapy-related myelodysplastic syndrome (t-MDS). T-AML/t-MDS occurs in 1-10\% of individuals who receive alkylator- or epipodophyllotoxin-based chemotherapy or radiation to treat a primary malignancy. ${ }^{6}$ For the seven individuals surveyed in this study, matched leukemia/ normal whole-genome sequencing identified the t-AML/t-MDSspecific somatic mutations present at diagnosis. We applied our method for ECS to identify leukemia-specific mutations in four individuals from DNA extracted from blood and bone marrow samples collected years before diagnosis. In a separate study into the role of TP53 mutations in t-AML/t-MDS leukemogenesis, this method was used to identify leukemia-associated mutations at low frequency in samples banked years before diagnosis. ${ }^{7}$ In two cases, subclones were identified below the $1 \%$ threshold of detection governed by conventional NGS. These results highlight the ability of targeted ECS to identify clinically silent single-nucleotide variations (SNVs).

We employed ECS by tagging individual DNA molecules with adapters containing $16 \mathrm{bp}$ random oligonucleotide molecular indexes in a manner similar to other reports. ${ }^{4,5,8}$ Our implementation of ECS easily targets loci of interest by single or multiplex PCR and inserts seamlessly into the standard NGS library preparation (Supplementary Figure 1, Supplementary Methods). Our only deviations from the standard protocol are ligation of customized adapters containing random indexes instead of the manufacturer's supplied adapters and a quantitative PCR (qPCR) quantification step before sequencing (Supplementary Table 1). Following sequencing, sequence reads containing the same index and originating from the same molecule are grouped into read families. Sequencing errors are identified by comparing reads within a read family and removed to create an error-corrected consensus sequence (ECCS). We performed a dilution series experiment to assess bias during library preparation and determine the limit of detection for ECS. For this experiment, we spiked DNA from a t-AML sample into control human DNA, which was serially diluted over five orders of magnitude. The experiment was comprised of two technical replicates targeting two separate mutations (20 total independent libraries). The results demonstrate that ECS is quantitative to a VAF of 1:10000 molecules and provides a highly reproducible digital readout of tumor DNA prevalence in a heterogeneous DNA sample $\left(r^{2}\right.$ of 0.9999 and 0.9991 , Figures 1a and b). We next characterized the error profile based on the wild-type nucleotides included in the dilution series experiment. Variant identification using the ECCSs was $99 \%$ specific at a VAF of 0.0016 versus 0.0140 for deep sequencing alone (Figure 1c). We noticed that ECCS errors were heavily biased towards $G$ to $T$ transversions and to a lesser degree $C$ to $T$ transitions (Figure 1d, Supplementary Figure 2), as previously observed. ${ }^{4,9}$ When separated by substitution type, variants identified from the ECCSs were $99 \%$ specific at a VAF of 0.0034 for $G$ to $T$ ( $C$ to $A$ ) mutations, 0.00020 for $C$ to $T$ ( $G$ to $A$ ) mutations and 0.000079 for the other eight possible substitutions. Although excess $G$ to $T$ mutations are a known consequence of DNA oxidation leading to 8 -oxo-guanine conversion, ${ }^{4}$ the pretreatment of samples with formamidopyrimidine-DNA glycosylase before PCR amplification did not appreciably improve the error profile of $G$ to T mutations (Supplementary Figure 3).

As proof of principle, we applied ECS to study rare pre-leukemic clonal hematopoiesis in seven individuals who later developed $\mathrm{t}$-AML/t-MDS. Leukemia/normal whole-genome sequencing at diagnosis was used to identify the leukemia-specific somatic mutations in each patient's malignancy (Supplementary Table 2). We applied targeted ECS to query these 18 different loci in 10 cryopreserved or formalin-fixed paraffin-embedded blood and bone marrow samples that were 9-22-year old and banked up to 12 years before diagnosis (Supplementary Table 3 ).

We generated $\sim 25 \mathrm{~Gb}$ of $150 \mathrm{bp}$ paired-end reads from six Illumina (San Diego, CA, USA) MiSeq runs. We targeted 1-7 somatic mutations per individual (25 mutations spanning $5.5 \mathrm{~kb}$ from 15 genes in total) and identified leukemia-specific subclonal populations in four individuals up to 12 years before diagnosis

Table 1. Patient-specific leukemia-associated somatic mutations identified by ECS

\begin{tabular}{|c|c|c|c|c|c|c|c|c|c|c|}
\hline UPN & Sample ID & Years prior & Gene & Chr & Position & Mut & Amino-acid change & Variant RFs & Reference RFs & $V A F$ \\
\hline \multirow[t]{2}{*}{446294} & 75.02 & 1 & OBSCN & 1 & 228461129 & A to $G$ & H1857R & 61238 & 156986 & 0.2806 \\
\hline & & & TP53 & 17 & 7578271 & $\mathrm{~T}$ to $\mathrm{A}$ & H193L & 220551 & 110047 & 0.6671 \\
\hline 499258 & 24.06 & 2 & RUNX1 & 21 & 36252865 & $C$ to $\mathrm{G}$ & R139P & 2 & 486196 & 0 \\
\hline 574214 & 26.04 & 7 & DMD & $\mathrm{X}$ & 32827676 & $G$ to $A$ & R187* & 7 & 199945 & 0 \\
\hline \multirow{4}{*}{643006} & 80.01 & 12 & ASXL1 & 20 & 31022448 & $\mathrm{G}$ to $\mathrm{T}$ & G645C & 7 & 85781 & 0.0001 \\
\hline & & & ASXL1 & 20 & 31022442 & del G & G645fs & 2898 & 82245 & 0.034 \\
\hline & & & GATA2 & 3 & 128200135 & del CTT & K390in_fr_del & 0 & 4187 & 0 \\
\hline & & & U2AF1 & 21 & 44524456 & $\mathrm{G}$ to $\mathrm{T}$ & S34Y & 85 & 414613 & 0.0002 \\
\hline \multirow[t]{6}{*}{684949} & 91.01 & 5 & ASXL1 & 20 & 31023112 & $T$ to $\mathrm{G}$ & L866* & 3583 & 853598 & 0.0042 \\
\hline & & & U2AF1 & 21 & 44524456 & $\mathrm{G}$ to $\mathrm{T}$ & S34Y & 545 & 514410 & 0.0011 \\
\hline & 92.02 & 4 & ASXL1 & 20 & 31023112 & $T$ to $\mathrm{G}$ & L866* & 54074 & 535976 & 0.0916 \\
\hline & & & U2AF1 & 21 & 44524456 & $\mathrm{G}$ to $\mathrm{T}$ & S34Y & 11195 & 355276 & 0.0305 \\
\hline & 93.01 & 3 & ASXL1 & 20 & 31023112 & $T$ to $G$ & L866* & 17319 & 573629 & 0.0293 \\
\hline & & & U2AF1 & 21 & 44524456 & $\mathrm{G}$ to $\mathrm{T}$ & S34Y & 827 & 92104 & 0.0089 \\
\hline \multirow[t]{7}{*}{856024} & 30.02 & 1 & S100A4 & 1 & 153517192 & A to $G$ & F27L & 0 & 211512 & 0 \\
\hline & & & IGSF8 & 1 & 160062252 & $G$ to $A$ & P516S & 0 & 22614 & 0 \\
\hline & & & PLA2R1 & 2 & 160798389 & A to $G$ & L1431P & 2 & 338616 & 0 \\
\hline & & & POU3F2 & 6 & 99282794 & $C$ to $A$ & S15R & 8 & 201240 & 0 \\
\hline & & & ANKRD18B & 9 & 33524645 & $G$ to $A$ & C53Y & 7 & 214836 & 0 \\
\hline & & & ESR2 & 14 & 64701847 & $G$ to $A$ & A416V & 10 & 135861 & 0.0001 \\
\hline & & & FBN3 & 19 & 8155081 & $G$ to $A$ & P2029L & 0 & 152304 & 0 \\
\hline \multirow[t]{4}{*}{942008} & 33.04 & 9 & IDH2 & 15 & 90631934 & $\mathrm{C}$ to $\mathrm{T}$ & R88Q & 23170 & 236587 & 0.0892 \\
\hline & & & RUNX1 & 21 & 36231791 & $\mathrm{~T}$ to $\mathrm{C}$ & D171G & 40 & 253168 & 0.0002 \\
\hline & 107.01 & $<1$ & $\mathrm{IDH} 2$ & 15 & 90631934 & $\mathrm{C}$ to $\mathrm{T}$ & R88Q & 138180 & 161371 & 0.4613 \\
\hline & & & RUNX1 & 21 & 36231791 & $\mathrm{~T}$ to $\mathrm{C}$ & D171G & 368438 & 50796 & 0.8788 \\
\hline
\end{tabular}

Abbreviations: $E C S$, error-corrected sequencing; RFs, read families; VAF, variant allele fraction. Two to seven mutations were queried per individual and the number of read families containing the variant allele or reference allele were reported and used to calculate the variant allele fraction. 
(Table 1). For each sequencing library, we tagged $\sim 2.5$ million locus-specific amplicons generated from genomic DNA using high-fidelity PCR with randomly indexed custom adapters. Sequencing errors were removed to create ECCSs as described above. Each ECCS was then aligned to the reference genome for variant calling (Supplementary Figure 1).

Using conventional deep sequencing, we detected t-AML/tMDS-specific mutations in prior banked samples at variant allele fractions between 0.03 and 0.87 (data not shown). In one individual (UPN 684949), deep sequencing alone was insufficient to distinguish known $A S X L 1$ and U2AF1 mutations from the sequencing errors in samples banked 5 and 3 years before t-MDS diagnosis, respectively (Figures $1 \mathrm{e}$ and $\mathrm{f}$ ). However, ECS identified the L866* nonsense mutation in ASXL1 at a VAF of 0.004 (Figure $1 \mathrm{~g}$ ) and the S34Y missense mutation in U2AF1 at a VAF of 0.009 (Figure $1 \mathrm{~h}$ ). In addition, ECS was able to temporally quantify these mutations from three pre-t-MDS samples banked yearly from 3 to 5 years before diagnosis (Supplementary Figures 4 and 5). In two cases (UPN643006 and UPN942008), only a subset of the variants identified at diagnosis were present in the prior banked sample (Table 1). Specifically, in the UPN643006 sample, banked 12 years before diagnosis, a single-nucleotide deletion in ASXL1 was present at VAF 0.03 . But, the $G$ to $T$ substitution in ASXL1, CTT deletion in GATA2 and $G$ to $T$ substitution in U2AF1 were not detectable in this prior banked sample.

Here we present a practical and clinically oriented application for targeted error-corrected NGS utilizing single molecule indexing. This method easily integrates into existing NGS library preparation protocols and enables the quantification of previously undetectable mutations in heterogeneous DNA samples. The only modification to the standard NGS library preparation is the replacement of the stock adapters with our randomly indexed adapters and the addition of a QPCR step before sequencing. The qPCR step limits the number of molecules sequenced, ensuring adequate coverage for each read family. With these two modifications, we achieve highly specific detection for rare mutations. The bioinformatics analysis is straightforward and does not require proprietary algorithms or tools (Supplementary Methods). Our results highlight the ability of this method to identify rare subclonal populations in a heterogeneous biological sample. As applied to t-AML/t-MDS, we show these previously undetectable mutations are present years before diagnosis and fluctuate in prevalence over time.

A clinical application of ECS is to quantify minimal residual disease (MRD). As the genomic characterization of leukemia becomes more readily available, identifying causative genetic lesions and rare therapy-resistant subclones will become increasingly useful for risk stratification, therapeutic selection and disease monitoring. Already, whole-genome sequencing of $A M L$ has demonstrated that nearly every case of AML harbors one or more somatic SNVs. ${ }^{10}$ These SNVs are more reliable clonal markers of malignancy than cell surface markers, which can change over time. Leveraging this information, conventional NGS was implemented retrospectively to detect MRD harboring leukemia-specific insertions/deletions (indels) as rare as $0.00001 \mathrm{VAF}$ in NPM $1^{11}$ and 0.0001 VAF in RUNX1. ${ }^{12}$ This was possible because indels are only rarely generated erroneously by NGS. Unfortunately, measuring rare leukemia-associated substitutions is limited owing to the relatively high error profile of conventional NGS. ${ }^{13}$ However, ECS can achieve the 1:10000 limit of detection featured by conventional MRD platforms. ${ }^{14}$ For patients whose leukemia lacks suitable markers for conventional MRD, ECS could offer an alternative with comparable sensitivity and specificity that is easy to implement in a clinical sequencing lab. Furthermore, the ability to multiplex targets for ECS enables the surveillance of known mutations and the simultaneous discovery of new somatic mutations. Ongoing work will directly compare gold-standard MRD methods with targeted ECS in patients with and without relapsed leukemia.

\section{CONFLICT OF INTEREST}

The authors declare no conflict of interest.

AL Young ${ }^{1,2}$, TN Wong $^{3}$, AEO Hughes ${ }^{1,2}$, SE Heath ${ }^{3}$, TJ Ley $^{3}$, DC Link ${ }^{3}$ and TE Druley ${ }^{1,2}$

${ }^{1}$ Department of Pediatrics, Division of Hematology and Oncology, Washington University School of Medicine, Saint Louis, MO, USA;

${ }^{2}$ Center for Genome Sciences and Systems Biology, Washington University School of Medicine, Saint Louis, MO, USA and ${ }^{3}$ Department of Medicine, Division of Oncology, Washington University School of Medicine, Saint Louis, MO, USA E-mail:Druley_t@kids.wustl.edu

\section{REFERENCES}

1 Holstege H, Pfeiffer W, Sie D, Hulsman M, Nicholas TJ, Lee CC et al. Somatic mutations found in the healthy blood compartment of a 115-yr-old woman demonstrate oligoclonal hematopoiesis. Genome Res 2014; 24: 733-742.

2 Walter MJ, Shen D, Ding L, Shao J, Koboldt DC, Chen K et al. Clonal architecture of secondary acute myeloid leukemia. N Engl J Med 2012; 366: 1090-1098.

3 Welch JS, Ley TJ, Link DC, Miller CA, Larson DE, Koboldt DC et al. The Origin and Evolution of Mutations in Acute Myeloid Leukemia. Cell 2012; 150: 264-278.

4 Schmitt MW, Kennedy SR, Salk JJ, Fox EJ, Hiatt JB, Loeb LA. Detection of ultra-rare mutations by next-generation sequencing. Proc Natl Acad Sci USA 2012; 109: 14508-14513.

5 Kinde I, Wu J, Papadopoulos N, Kinzler KW, Vogelstein B. Detection and quantification of rare mutations with massively parallel sequencing. Proc Natl Acad Sci USA 2011; 108: 9530-9535.

6 Godley LA, Larson RA. Therapy-related myeloid leukemia. Semin Oncol 2008; 35: 418-429.

7 Wong T, Ramsingh G, Young AL, Miller CA, Touma W, Welch JS et al. The role of TP53 mutations in the origin and evolution of therapy-related AML. Nature 2015; 518: $552-555$.

8 Fu GK, Xu W, Wilhelmy J, Mindrinos MN, Davis RW, Xiao W et al. Molecular indexing enables quantitative targeted RNA sequencing and reveals poor efficiencies in standard library preparations. Proc Natl Acad Sci USA 2014; 111: 1891-1896.

9 Lou DI, Hussmann Ja, McBee RM, Acevedo A, Andino R, Press WH et al. High-throughput DNA sequencing errors are reduced by orders of magnitude using circle sequencing. Proc Natl Acad Sci USA 2013; 110: 19872-19877.

10 Cancer Genome Atlas Research Network. Genomic and epigenomic landscapes of adult de novo acute myeloid leukemia. N Engl J Med 2013; 368: 2059-2074.

11 Salipante SJ, Fromm JR, Shendure J, Wood BL, Wu D. Detection of minimal residual disease in NPM1-mutated acute myeloid leukemia by next-generation sequencing. Mod Pathol 2014; 27: 1438-1446.

12 Kohlmann a, Nadarajah N, Alpermann T, Grossmann V, Schindela S, Dicker F et al. Monitoring of residual disease by next-generation deep-sequencing of RUNX1 mutations can identify acute myeloid leukemia patients with resistant disease. Leukemia 2014; 28: 129-137.

13 Loman NJ, Misra RV, Dallman TJ, Constantinidou C, Gharbia SE, Wain J et al. Performance comparison of benchtop high-throughput sequencing platforms. Nat Biotechnol 2012; 30: 434-439.

14 Hourigan CS, Karp JE. Minimal residual disease in acute myeloid leukaemia. Nat Rev Clin Oncol 2013; 10: 460-471.

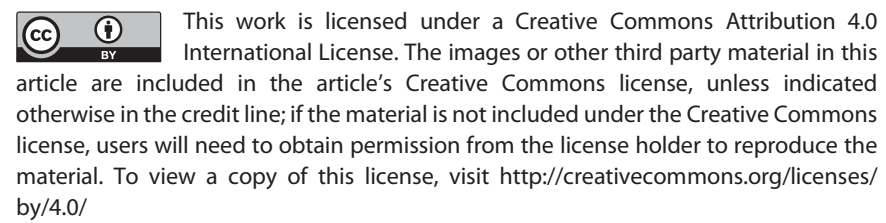
by/4.0/ 